\title{
Efficacité des pratiques en matière d'hygiène des mains dans la prévention de l'infection par le virus de la grippe en milieu communautaire : examen systématique
}

\author{
K Moncion'1, K Young1', M Tunis ${ }^{1}$, S Rempel'1, R Stirling1', L Zhao'*
}

\section{Résumé}

Contexte : L'hygiène des mains est une mesure reconnue et efficace dans la prévention et la lutte contre les infections dans les établissements de soins de santé. Toutefois, l'efficacité des pratiques d'hygiène des mains dans la prévention de la grippe et de sa transmission en milieu communautaire n'est pas clairement établie.

Objectif : Repérer, examiner et synthétiser les données disponibles sur l'efficacité de l'hygiène des mains dans la prévention de la grippe présumée ou confirmée en laboratoire et sa transmission en milieu communautaire.

Méthodologie : Un protocole d'examen systématique des données a été établi avant le début de l'examen. Trois bases de données électroniques (MEDLINE, Embase et la Bibliothèque Cochrane) ont été consultées pour repérer les études pertinentes. Deux examinateurs ont filtré séparément les titres, les résumés et les textes intégraux des études à partir des bases de données interrogées. L'extraction des données et l'évaluation de la qualité des études retenues ont été effectuées par un seul examinateur puis validées par un second examinateur. Les études retenues ont été synthétisées et analysées de manière narrative.

Résultats : Au total, 16 études ont été retenues aux fins de l'examen. Les études étaient de faible qualité méthodologique, et on a constaté une forte variabilité des protocoles, des milieux, du contexte et des indicateurs de recherche. Neuf études ont évalué l'efficacité des interventions ou des pratiques d'hygiène des mains pour la prévention des cas de grippe présumés ou confirmés en laboratoire dans le milieu communautaire; une différence significative a été constatée dans six études, tandis que trois études n'ont pas montré une telle différence. Sept études ont évalué l'efficacité des pratiques d'hygiène des mains pour la prévention de la transmission de la grippe présumée ou confirmée en laboratoire en milieu communautaire; une différence significative a été notée dans deux études, tandis que cinq études n'ont pas montré une telle différence.

Conclusion : L'efficacité de l'hygiène des mains contre l'infection par le virus de la grippe et sa transmission en milieu communautaire est difficile à déterminer à partir des données disponibles. Considérant que l'efficacité des pratiques d'hygiène des mains a été éprouvée dans d'autres contextes, il n'existe aucune donnée probante indiquant qu'il faut cesser ces pratiques pour réduire le risque d'infection par le virus de la grippe et sa transmission en milieu communautaire.
Citation proposée : Moncion K, Young K, Tunis M, Rempel S, Stirling R, Zhao L. Efficacité des pratiques en matière d'hygiène des mains dans la prévention de l'infection par le virus de la grippe en milieu communautaire : examen systématique. Relevé des maladies transmissibles au Canada 2019;45(1):13-25. https://doi.org/10.14745/ ccdr.v45i01a02f

Mots-clés : communauté, hygiène des mains, désinfectant pour les mains, lavage des mains, grippe, transmission de la grippe, examen systématique

Page 13 RMTC • le 3 janvier, $2019 \bullet$ Volume 45-1
Cette œuvre est mise à la disposition selon les termes de la licence internationale Creative Commons Attribution 4.0

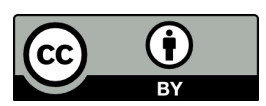

Affiliation

${ }^{1}$ Centre de l'immunisation et des maladies respiratoires infectieuses, Agence de la santé publique du Canada, Ottawa (Ontario)

*Correspondance: linlu.zhao@ canada.ca 


\section{Introduction}

L'hygiène des mains est une modalité de prévention et de contrôle des infections fréquemment recommandée pour réduire le risque de grippe et de transmission de la grippe en milieu communautaire et dans les établissements de soins de santé. Les protocoles d'hygiène de base des mains par lavage des mains au savon et à l'eau courante (1) et/ou frottage des mains avec un désinfectant à base d'alcool $(1,2)$ sont efficaces pour l'élimination physique du virus de la grippe sur les mains chez l'humain.

Les pratiques d'hygiène des mains se sont révélées efficaces pour réduire l'infection et la transmission des agents pathogènes associés aux soins dans les établissements de soins de santé (3), pour réduire les affections gastro-intestinales et respiratoires dues à un pathogène non-spécifique en milieu communautaire $(4,5)$, et pour désinfecter, éliminer les contaminants et réduire l'incidence des infections nosocomiales dans les établissements de soins de santé (3).

Un aspect qui a été moins souvent étudié est le degré de protection qu'offrent les pratiques d'hygiène des mains contre l'infection par le virus de la grippe et sa transmission en milieu communautaire. Une recherche documentaire initiale a permis de repérer deux examens systématiques ayant mené à des conclusions différentes. Un examen des essais contrôlés randomisés a conclu que l'hygiène des mains associée au port d'un masque facial en milieu communautaire était efficace contre la grippe confirmée en laboratoire ou le syndrome grippal, mais que l'hygiène des mains seule ne l'était pas (6). Dans un autre examen des essais d'intervention et des études observationnelles, on a constaté une réduction des cas de grippe associée aux interventions d'hygiène des mains dans les écoles, mais aucun effet sur la transmission secondaire de la grippe dans les ménages au sein des communautés où il y avait déjà un cas index (7).

Un examen systématique a été effectué pour repérer, examiner et synthétiser les plus récentes données sur l'efficacité de I'hygiène des mains en tant qu'intervention dans la prévention de la grippe présumée ou confirmée en laboratoire et de sa transmission en milieu communautaire. Le terme " grippe présumée » désigne les cas non confirmés en laboratoire, y compris le syndrome grippal et les affections respiratoires aiguës.

\section{Méthodologie}

Les paramètres de l'examen systématique, la stratégie de recherche et le plan d'analyse ont été établis avant l'examen. Le terme "hygiène des mains " désigne le lavage des mains, le lavage avec un antiseptique et la prise de mesures visant à maintenir la santé des mains et des ongles (8). La stratégie de recherche (annexe 1) a été mise au point en collaboration avec un bibliothécaire de recherche. Les recherches ont été effectuées dans les bases de données électroniques MEDLINE, Embase et la Bibliothèque Cochrane, depuis leur création jusqu'au 5 juin 2017, au moyen de termes associés à la grippe et à l'hygiène des mains. Les recherches ont été limitées aux articles publiés en anglais ou en français.

Les études retenues aux fins d'examen devaient satisfaire aux critères suivants :

- Étude effectuée en milieu communautaire, défini comme un contexte différent d'un établissement de soins de santé, ouvert et sans confinement et où aucun soin particulier n'est offert aux participants (p. ex. école, lieu de travail, ménage) (6)

- Étude observationnelle ayant évalué I'hygiène des mains en tant qu'exposition d'intérêt (p. ex. pratique d'hygiène des mains observée ou déclarée), ou essai clinique associant mesures éducatives, promotion et mise à disposition de produits relatifs à l'hygiène des mains, mais ayant évalué une intervention d'hygiène des mains raisonnablement susceptible d'exercer une influence indépendante

- Étude ayant évalué les répercussions de l'hygiène des mains sur :

- la grippe présumée ou infection confirmée en laboratoire; ou

- la transmission de la grippe présumée ou confirmée en laboratoire

Toute étude remplissant un ou plusieurs des critères suivants était exclue :

- Étude réalisée uniquement dans des établissements de soins de santé

- Étude ayant évalué une intervention à plusieurs volets pour laquelle on ne pouvait pas raisonnablement s'attendre à ce que l'hygiène des mains exerce une influence indépendante

- Étude qui n'est pas une recherche clinique (p. ex. examen de la documentation scientifique, éditorial, article d'opinion ou de nouvelles, étude in vitro ou ne portant pas sur des humains)

La sélection des études a été effectuée de manière indépendante par deux examinateurs. Les listes de références des études retenues et les articles de recherche secondaires pertinents repérés dans le cadre de la recherche ont également été dépouillés pour trouver des publications pertinentes. Un premier examinateur (KM) a effectué l'extraction des données et l'évaluation de la qualité, tandis qu'un second examinateur (LZ) s'est chargé de la validation. On a extrait les données concernant le protocole d'étude, la population, le contexte, l'intervention $d$ 'hygiène des mains ( $p$. ex. dans les essais cliniques) ou 
les pratiques d'hygiène des mains (p. ex. dans les études observationnelles) ainsi que les résultats d'intérêt. La qualité des études a été évaluée au moyen de l'Outil d'évaluation du risque de biais de la Collaboration Cochrane pour les essais cliniques randomisés (ECR) (9) et de l'outil d'évaluation de la qualité du Projet de pratique efficace en santé publique pour les études observationnelles (10). En cas de désaccord, les deux évaluateurs devaient discuter du cas afin de parvenir à un consensus.

Une synthèse et une analyse descriptive des données ont été prévues afin de résumer la direction, la taille et la signification statistique des estimations des effets signalés de divers paramètres définis dans les études, et pour explorer les tendances générales se dégageant des données extraites des études retenues. Lorsque cela était possible, des méta-analyses ont été prévues pour évaluer le lien entre l'hygiène des mains et les résultats relatifs à la grippe selon le niveau de revenus du pays de l'étude, le protocole de l'étude, le contexte, l'intervention évaluée et le paramètre analysé.

\section{Résultats}

Après avoir fait une recherche dans les bases de données suivie d'une recherche manuelle et après avoir éliminé les doublons, il restait 998 documents. Parmi ces derniers, 115 documents ont été sélectionnés aux fins de l'examen du texte intégral. Lorsque tous les critères d'inclusion et d'exclusion ont été appliqués, il restait 16 études évaluables, dont sept ECR et neuf études observationnelles. La figure 1 résume le processus de sélection de l'étude.

Les ECR évalués avec l'outil d'évaluation du risque de biais de la Collaboration Cochrane comportaient tous un risque élevé de biais (11-17). Lorsqu'on a évalué les études observationnelles avec l'outil d'évaluation de la qualité du Projet de pratique efficace en santé publique, on a constaté que sept études observationnelles sur neuf étaient de faible qualité (18-24) et que deux étaient de qualité moyenne $(25,26)$. Les examinateurs ont décidé par après de ne pas effectuer de méta-analyse, car le nombre d'études incluses n'était pas adéquat pour leur regroupement en fonction des caractéristiques d'intérêt.

\section{ECR sur des interventions d'hygiène des mains}

Sur les sept ECR inclus, six ont évalué la mise à disposition d'un désinfectant pour les mains ou d'un savon assortis d'un mode d'emploi $(11-14,16,17)$. Dans un ECR, on a effectué une intervention en ligne visant à diffuser de l'information et à promouvoir le lavage des mains, sans mettre du désinfectant ou du savon à la disposition des participants (15). Aucun ECR ne donnait suffisamment de détails concernant les instructions ou l'information offertes aux participants sur le lavage ou l'antisepsie des mains permettant de comparer ces interventions aux pratiques exemplaires.
Figure 1 : Diagramme du processus de sélection des études

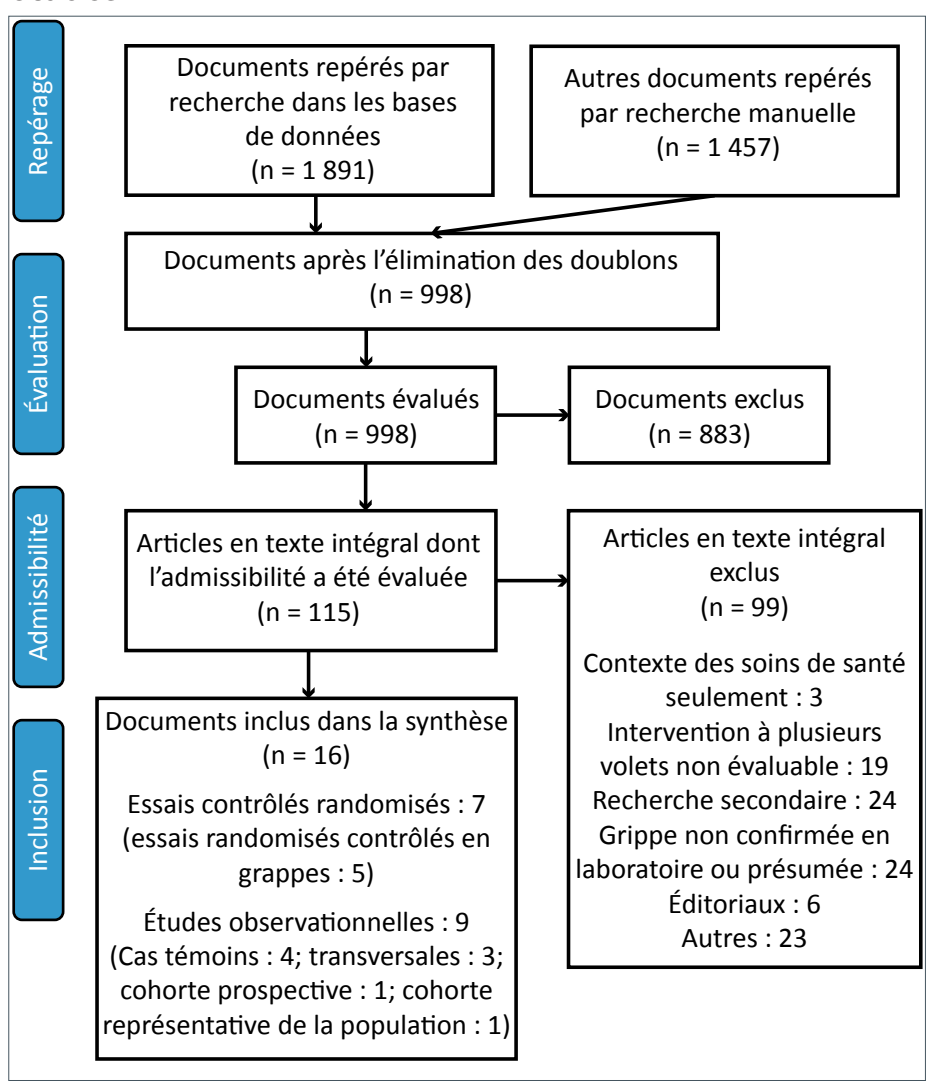

Abréviation : $\mathrm{n}=$ nombre

\section{Études observationnelles sur les pratiques d'hygiène des mains}

Dans quatre des neuf études observationnelles incluses, la fréquence de lavage des mains a été autodéclarée $(21,24-26)$. Parmi les cinq études restantes, on a dichotomisé les comportements de lavage des mains observés en " observés " ou "non observés » dans une étude (18) et en "fréquents" et "peu fréquents » dans une autre (19). Ces études ne comportaient aucune définition ni mention de critères relatifs au lavage des mains qui auraient permis d'estimer la fréquence du lavage des mains ou le dénombrement des événements de lavage des mains. Deux études ont évalué la qualité de la pratique d'hygiène des mains auto-déclarée, c'est-à-dire qu'elle était « bonne " ou " mauvaise » (20) et " optimale " ou " sous-optimale » (23). Une de ces études définissait la pratique $d$ 'hygiène des mains optimale dans les mêmes termes que les pratiques exemplaires publiées (20). Dans une autre étude, on a noté les déclarations des sujets concernant l'adoption de diverses interventions non pharmacologiques, dont le lavage plus fréquent des mains et l'usage de désinfectant pour les mains (22). 


\section{Hygiène des mains et grippe}

Neuf études ont évalué l'efficacité des interventions ou des pratiques d'hygiène des mains dans la prévention de la grippe présumée ou confirmée en laboratoire en milieu communautaire, dont deux ECR $(13,15)$, une étude de cohortes $(25)$, trois études cas témoins $(19,21,26)$ et trois études transversales $(18,20,23)$.

Les résultats des études étaient variables; six études sur neuf ont conclu qu'une intervention ou pratique d'hygiène des mains réduisait le nombre de cas de grippe confirmée en laboratoire $(21,26)$ ou présumée $(15,18,20,23)$, tandis que trois études ont conclu qu'il n'y avait pas de lien statistiquement significatif entre l'hygiène des mains et une diminution des cas de grippe $(13,19,25)$. Un des deux ECR a fait état d'un lien significatif entre le lavage des mains et une diminution du risque de syndrome grippal (15), tandis que l'autre ECR n'a relevé aucun effet d'une intervention de désinfection des mains sur le lieu de travail sur les cas de grippe cliniquement diagnostiquée auto-déclarée (13). Quant aux études observationnelles, fondées sur les pratiques d'hygiène des mains auto-déclarées $(20,21,23,25,26)$ ou observées $(18,19)$, elles ont pour la plupart fait état d'une probabilité d'infection présumée significativement inférieure sur le plan statistique $(18,20,21,23,26)$. II n'a pas été possible de réaliser une analyse qualitative plus détaillée des résultats étant donné le nombre limité d'études hétérogènes. Les résultats sont résumés dans le tableau 1.

\section{Hygiène des mains et transmission de la grippe}

Sept études ont évalué l'efficacité des pratiques d'hygiène des mains pour prévenir la transmission de la grippe présumée ou confirmée en laboratoire en milieu communautaire, dont cinq $\operatorname{ECR}(11,12,14,16,17)$, une étude de cohortes (22) et une étude cas témoins (24). Dans la majorité de ces études, on a évalué la transmission de la grippe en milieu communautaire en estimant le taux d'attaques secondaires (TAS) dans les ménages (p. ex. proportion de personnes prédisposées qui deviennent malades) quant à la grippe présumée ou confirmée en laboratoire $(11,12,14,16,17)$.

Dans cinq études sur sept, on n'a établi aucun lien statistiquement significatif entre l'intervention ou la

Tableau 1 : Résumé des données relatives à l'efficacité des pratiques d'hygiène des mains dans la prévention de la grippe présumée ou confirmée en laboratoire en milieu communautaire

\begin{tabular}{|c|c|c|c|c|}
\hline Étude & $\begin{array}{c}\text { Taille de } \\
\text { l'échantillon (n) }\end{array}$ & $\begin{array}{l}\text { Intervention d'hygiène des } \\
\text { mains ou pratique déclarée/ } \\
\text { intervention témoin }\end{array}$ & $\begin{array}{l}\text { Principal } \\
\text { paramètre } \\
\text { d'évaluation }\end{array}$ & Principaux résultats pertinents \\
\hline \multicolumn{5}{|c|}{ Essai contrôlé randomisé } \\
\hline $\begin{array}{l}\text { Hubner et al., } 2010 \\
\text { (13) }\end{array}$ & $\begin{array}{l}134 \\
\text { (intervention : } \\
\text { 67; intervention } \\
\text { témoin : 67) }\end{array}$ & $\begin{array}{l}\text { Instruction d'utiliser un désinfectant } \\
\text { pour les mains à base d'alcool au } \\
\text { moins cinq fois par jour seulement } \\
\text { au travail; désinfectant fourni } \\
\text { Intervention témoin : Pas } \\
\text { d'instructions ni de désinfectant } \\
\text { fournis }\end{array}$ & $\begin{array}{l}\text { Auto-déclaration } \\
\text { de la grippe } \\
\text { cliniquement } \\
\text { diagnostiquée }\end{array}$ & $\begin{array}{l}\text { Aucune différence dans la probabilité de } \\
\text { grippe cliniquement diagnostiquée entre les } \\
\text { groupes d'intervention et témoin } \\
\text { (RC:1,02; IC à } 95 \%: 0,20-5,23)\end{array}$ \\
\hline Little et al., 2015 (15) & $\begin{array}{l}20066 \\
\text { (intervention : } \\
10040 ; \\
\text { intervention } \\
\text { témoin : } 10026 \text { ) }\end{array}$ & $\begin{array}{l}\text { Accès à une intervention en ligne } \\
\text { fournissant de l'information sur } \\
\text { l'importance de la grippe et le rôle } \\
\text { du lavage des mains } \\
\text { Intervention témoin: Pas d'accès à } \\
\text { l'intervention en ligne }\end{array}$ & Syndrome grippal & $\begin{array}{l}\text { Participants du groupe d'intervention : } \\
\text { diminution du risque de syndrome grippal } \\
\text { déclaré au cours des quatre derniers mois } \\
\text { (RTa : 0,80; IC à } 95 \%: 0,72-0,92) \text { et du } \\
\text { dernier mois (RTa : 0,85; IC à } 95 \% \text { : } \\
0,77-0,94) \text { par rapport au groupe témoin }\end{array}$ \\
\hline \multicolumn{5}{|l|}{ Étude de cohortes } \\
\hline $\begin{array}{l}\text { Merk et al., } 2004 \\
\text { (25) }\end{array}$ & 4365 & $\begin{array}{l}\text { Auto-déclaration de la fréquence } \\
\text { du lavage des mains }\end{array}$ & $\begin{array}{l}\text { Syndrome grippal } \\
\text { et ARA auto- } \\
\text { déclarés }\end{array}$ & $\begin{array}{l}\text { Aucune différence statistiquement } \\
\text { significative entre les adultes qui se lavaient } \\
\text { les mains } \geq 5 \text { fois par jour et ceux qui se } \\
\text { lavaient les mains } 2 \text { à } 4 \text { fois par jour quant à } \\
\text { l'incidence du syndrome grippal (RTa : } 1,10 \text { - } \\
1,48 \text { ) et des ARA (RTa : } 1,08-1,22)\end{array}$ \\
\hline \multicolumn{5}{|l|}{ Étude cas témoins } \\
\hline $\begin{array}{l}\text { Doshi et al., } 2015 \\
\text { (19) }\end{array}$ & $\begin{array}{l}486 \text { (cas : } 145 ; \\
\text { témoins : } 341 \text { ) }\end{array}$ & $\begin{array}{l}\text { Observation du lavage des mains } \\
\text { dans le ménage (fréquent/peu } \\
\text { fréquent) }\end{array}$ & $\begin{array}{l}\text { Grippe confirmée } \\
\text { en laboratoire }\end{array}$ & $\begin{array}{l}\text { Pas de lien statistiquement significatif } \\
\text { entre le lavage des mains avec de l'eau et } \\
\text { du savon dans les ménages et la grippe } \\
\text { confirmée en laboratoire (RCa : } 1,06 \text {; IC à } 95 \\
\%: 0,90-1,24)\end{array}$ \\
\hline Liu et al., 2016 (21) & $\begin{array}{l}200 \text { (cas : } 100 ; \\
\text { témoins : } 100 \text { ) }\end{array}$ & $\begin{array}{l}\text { Auto-déclaration de la fréquence } \\
\text { du lavage des mains }\end{array}$ & $\begin{array}{l}\text { Grippe confirmée } \\
\text { en laboratoire }\end{array}$ & $\begin{array}{l}\text { Diminution statistiquement significative } \\
\text { de la probabilité de grippe confirmée en } \\
\text { laboratoire avec le lavage des mains (de } 54 \\
\% \text { par paliers d'augmentation d'une unité du } \\
\text { score de lavage des mains; RCa : } 0,46 \text {; IC à } \\
95 \%: 0,29-0,74 \text { ) }\end{array}$ \\
\hline
\end{tabular}


Tableau 1 (suite): Résumé des données relatives à l'efficacité des pratiques d'hygiène des mains dans la prévention de la grippe présumée ou confirmée en laboratoire en milieu communautaire

\begin{tabular}{|c|c|c|c|c|}
\hline \multicolumn{5}{|c|}{ Étude cas témoins (suite) } \\
\hline $\begin{array}{l}\text { Torner et al., } 2015 \\
\text { (26) }\end{array}$ & $\begin{array}{l}478 \text { (cas : } 239 ; \\
\text { témoins : } 239 \text { ) }\end{array}$ & $\begin{array}{l}\text { Auto-déclaration de la fréquence } \\
\text { du lavage des mains }\end{array}$ & $\begin{array}{l}\text { Grippe confirmée } \\
\text { en laboratoire }\end{array}$ & $\begin{array}{l}\text { Probabilité de grippe confirmée en } \\
\text { laboratoire significativement plus faible sur } \\
\text { le plan statistique chez les enfants qui ont } \\
\text { déclaré se laver les mains } \geq 5 \text { fois par jour } \\
\text { (RCa : } 0,62 ; \text { IC à } 95 \%: 0,39-0,99)\end{array}$ \\
\hline & & & & $\begin{array}{l}\text { Aucun lien statistiquement significatif } \\
\text { entre la grippe confirmée en laboratoire et } \\
\text { l'emploi d'un désinfectant pour les mains à } \\
\text { base d'alcool (RCa : } 1,54 ; \text { IC à } 95 \%: 0,8- \\
2,66 \text { ) ou le lavage des mains après contact } \\
\text { avec des surfaces contaminées (RCa : } 0,62 \text {; } \\
\text { IC à } 95 \%: 0,29-1,31 \text { ) }\end{array}$ \\
\hline \multicolumn{5}{|l|}{ Étude transversale } \\
\hline $\begin{array}{l}\text { Adesanya et al., } \\
2016(18)\end{array}$ & 28596 & $\begin{array}{l}\text { Observation du lavage des mains } \\
\text { (observé/non observé) }\end{array}$ & $\begin{array}{l}\text { Déclaration d'une } \\
\text { ARA par un parent }\end{array}$ & $\begin{array}{l}\text { Probabilité accrue de symptômes d'ARA } \\
\text { chez les enfants qui ne se lavaient pas les } \\
\text { mains par rapport aux enfants qui se lavaient } \\
\text { les mains (RCa: } 1,66 \text {; IC à } 95 \%: 1,33-2,07 \text { ) }\end{array}$ \\
\hline $\begin{array}{l}\text { Hashim et al., } 2016 \\
\text { (20) }\end{array}$ & 468 & $\begin{array}{l}\text { Auto-déclaration de la pratique } \\
\text { d'hygiène des mains (bonne/ } \\
\text { mauvaise) }\end{array}$ & $\begin{array}{l}\text { Affection } \\
\text { respiratoire } \\
\text { auto-déclarée } \\
\text { (syndrome grippal } \\
\text { et autre que le } \\
\text { syndrome grippal) }\end{array}$ & $\begin{array}{l}\text { Chez les pèlerins du hadj qui ont déclaré } \\
\text { de bonnes pratiques d'hygiène des mains, } \\
\text { la probabilité de présenter une affection } \\
\text { respiratoire était significativement inférieure } \\
\text { sur le pan statistique que celle observée } \\
\text { chez ceux qui n'ont pas déclaré de bonnes } \\
\text { pratiques d'hygiène des mains (RC : } 0,41 \text {; IC } \\
\text { à } 95 \%: 0,20-0,85 \text { ) }\end{array}$ \\
\hline Wu et al., 2016 (23) & 13003 & $\begin{array}{l}\text { Auto-déclaration du lavage } \\
\text { des mains ou de l'emploi d'un } \\
\text { désinfectant pour les mains } \\
\text { (optimal/sous-optimal) }\end{array}$ & $\begin{array}{l}\text { Auto-déclaration } \\
\text { d'un syndrome } \\
\text { grippal }\end{array}$ & $\begin{array}{l}\text { Lien statistiquement significatif entre une } \\
\text { hygiène des mains optimale (définition non } \\
\text { fournie) et une probabilité plus faible de } \\
\text { déclarer un syndrome grippal (RC: } 0,87 \text {; IC à } \\
95 \%: 0,80-0,94 \text { ) }\end{array}$ \\
\hline
\end{tabular}

Abréviations : ARA, affection respiratoire aiguë; IC, intervalle de confiance; n, nombre; RC, rapport de cotes; RCa, rapport de cotes ajusté; $\mathrm{RTa}$, rapport de taux ajusté; $\geq$, au moins

pratique d'hygiène des mains et la transmission de la grippe $(11,12,14,16,22)$. Dans un ECR, on a observé une différence statistiquement significative quant aux TAS liés au syndrome grippal entre le lavage des mains, le lavage des mains avec port du masque facial et les interventions témoins $(0,17,0,18$ et 0,09 , respectivement), mais pas quant aux TAS liés à la grippe confirmée en laboratoire (17). Une étude cas témoins a mis en évidence un lien statistiquement significatif entre le lavage des mains au moins trois fois par jour et la réduction de la probabilité de transmission du virus de la grippe A pandémique (H1N1) dans les ménages (24).

Dans quatre ECR par grappes effectués sur des ménages, l'intervention d'hygiène des mains a été mise en œuvre après le repérage du cas index $(11,12,16,17)$. Deux de ces quatre études ont évalué un sous-groupe de ménages où l'intervention a été mise en œuvre pendant une période définie après l'apparition des symptômes dans le cas index (p. ex. moins de 36 ou 48 heures). Une des deux études n'a pas relevé de différence statistiquement significative entre le groupe pratiquant l'hygiène des mains et le groupe témoin (12), alors que l'autre étude a noté des résultats variables selon la méthode d'établissement de la grippe et du type de grippe (17). Quatre ECR par grappes sur cinq n'ont pas relevé de différence statistiquement significative quant aux TAS liés la grippe présumée ou confirmée en laboratoire entre le groupe pratiquant l'hygiène des mains et le groupe témoin $(11,12,14,16)$, tandis que le dernier a obtenu des résultats variables selon le paramètre évalué (17). Le tableau 2 présente un aperçu des résultats. 
Tableau 2 : Résumé des données relatives à l'efficacité des pratiques d'hygiène des mains dans la prévention de la transmission de la grippe présumée ou confirmée en laboratoire en milieu communautaire

\begin{tabular}{|c|c|c|c|c|}
\hline Étude & $\begin{array}{c}\text { Taille de } \\
\text { l'échantillon (n) }\end{array}$ & $\begin{array}{l}\text { Intervention d'hygiène des mains } \\
\text { ou pratique déclarée/ } \\
\text { intervention témoin }\end{array}$ & $\begin{array}{l}\text { Principal } \\
\text { paramètre } \\
\text { d'évaluation }\end{array}$ & Principaux résultats pertinents \\
\hline \multicolumn{5}{|c|}{ Essai contrôlé randomisé } \\
\hline $\begin{array}{l}\text { Cowling et al., } \\
2008(11)\end{array}$ & $\begin{array}{l}198 \text { ménages } \\
\text { (hygiène des } \\
\text { mains : } 36 ; \\
\text { masque facial : 35; } \\
\text { intervention } \\
\text { témoin : } 127 \text { ) }\end{array}$ & $\begin{array}{l}\text { Intervention d'hygiène des } \\
\text { mains : Même information que dans } \\
\text { l'intervention témoin, plus information } \\
\text { sur l'hygiène des mains (efficacité } \\
\text { potentielle d'une hygiène adéquate } \\
\text { des mains pour réduire la transmission, } \\
\text { avec instructions) et distribution d'un } \\
\text { désinfectant pour les mains et de savon } \\
\text { Intervention avec masque facial : Même } \\
\text { information que dans l'intervention } \\
\text { témoin, plus information sur le masque } \\
\text { facial et distribution d'un masque facial à } \\
\text { chaque membre du ménage } \\
\text { Groupe témoin : Information sur une } \\
\text { alimentation et un mode de vie sains en } \\
\text { rapport avec la prévention des maladies } \\
\text { liées aux contacts du ménage et avec } \\
\text { le soulagement des symptômes pour le } \\
\text { sujet index }\end{array}$ & $\begin{array}{l}\text { TAS liés à la } \\
\text { grippe clinique } \\
\text { (trois définitions) } \\
\text { ou confirmée en } \\
\text { laboratoire }\end{array}$ & $\begin{array}{l}\text { Il n'y avait pas de différence } \\
\text { statistiquement significative entre les } \\
\text { groupes d'intervention quant aux TAS } \\
\text { liés à la grippe clinique ou confirmée en } \\
\text { laboratoire. La probabilité d'infection } \\
\text { secondaire par un contact du ménage } \\
\text { était statistiquement similaire pour le } \\
\text { groupe d'intervention d'hygiène des } \\
\text { mains et le groupe témoin quant à } \\
\text { la grippe clinique (RC: } 0,80-0,86) \text { et } \\
\text { confirmée en laboratoire }(R C: 1,07)\end{array}$ \\
\hline $\begin{array}{l}\text { Cowling et al., } \\
2009 \text { (12) }\end{array}$ & $\begin{array}{l}407 \text { ménages } \\
\text { (hygiène des } \\
\text { mains : } 136 ; \\
\text { hygiène des mains } \\
\text { et masque } \\
\text { facial : } 137 ; \\
\text { intervention } \\
\text { témoin : } 134 \text { ) }\end{array}$ & $\begin{array}{l}\text { Intervention d'hygiène des mains } \\
: \text { Même information que dans } \\
\text { l'intervention témoin, plus information } \\
\text { sur l'hygiène des mains (efficacité } \\
\text { potentielle d'une hygiène adéquate } \\
\text { des mains pour réduire la transmission, } \\
\text { avec instructions) et distribution d'un } \\
\text { désinfectant pour les mains et de savon } \\
\text { Intervention d'hygiène des mains et } \\
\text { masque facial : Même information } \\
\text { que dans l'intervention témoin et } \\
\text { l'intervention d'hygiène des mains, } \\
\text { plus information sur le masque facial et } \\
\text { distribution d'un masque facial à chaque } \\
\text { membre du ménage } \\
\text { Groupe témoin : Information sur une } \\
\text { alimentation et un mode de vie sains en } \\
\text { rapport avec la prévention des maladies } \\
\text { liées aux contacts du ménage et avec } \\
\text { le soulagement des symptômes pour le } \\
\text { sujet index }\end{array}$ & $\begin{array}{l}\text { TAS liés à la } \\
\text { grippe clinique } \\
\text { (deux définitions) } \\
\text { ou confirmée en } \\
\text { laboratoire }\end{array}$ & $\begin{array}{l}\text { Il n'y avait pas de différence } \\
\text { statistiquement significative entre les } \\
\text { groupes d'intervention quant aux TAS } \\
\text { liés aux cas secondaires cliniques ou } \\
\text { confirmés en laboratoire. La probabilité } \\
\text { d'une infection secondaire chez un } \\
\text { contact du ménage était statistiquement } \\
\text { similaire lorsqu'on comparait le groupe } \\
\text { d'intervention d'hygiène des mains quant } \\
\text { à la grippe clinique (RC }: 0,92-0,81) \text { ou } \\
\text { confirmée en laboratoire (RC : } 0,57) \text { et } \\
\text { le groupe d'intervention d'hygiène des } \\
\text { mains et portant un masque facial quant } \\
\text { à la grippe clinique (RC: } 1,25-1,68) \text { ou } \\
\text { confirmée en laboratoire }(R C: 0,77) \text { au } \\
\text { groupe témoin }\end{array}$ \\
\hline $\begin{array}{l}\text { Larson et al., } 2010 \\
\text { (14) }\end{array}$ & $\begin{array}{l}509 \text { ménages } \\
\text { (désinfectant } \\
\text { pour les mains : } \\
169 ; \text { désinfectant } \\
\text { pour les mains et } \\
\text { masque facial : 166; } \\
\text { intervention } \\
\text { témoin : 174) }\end{array}$ & $\begin{array}{l}\text { Intervention avec désinfectant pour } \\
\text { les mains: Matériel informatif et } \\
\text { désinfectant pour les mains emportés } \\
\text { par les membres du ménage à leur } \\
\text { milieu de travail ou à l'école } \\
\text { Intervention avec désinfectant pour } \\
\text { les mains et masque facial : Matériel } \\
\text { informatif, désinfectant pour les mains, } \\
\text { masque facial et mode d'emploi du } \\
\text { masque facial } \\
\text { Intervention témoin: Matériel informatif } \\
\text { sur la prévention et le traitement des } \\
\text { IVRS et de la grippe }\end{array}$ & $\begin{array}{l}\text { Syndrome } \\
\text { grippal et grippe } \\
\text { confirmée en } \\
\text { laboratoire } \\
\text { TAS liés aux IVRS, } \\
\text { au syndrome } \\
\text { grippal et à la } \\
\text { grippe confirmée } \\
\text { en laboratoire }\end{array}$ & $\begin{array}{l}\text { Aucune différence dans les taux de } \\
\text { syndrome grippal ou de grippe confirmée } \\
\text { en laboratoire entre les groupes } \\
\text { d'intervention et témoin. } \\
\text { Les TAS liés aux IVRS, au syndrome } \\
\text { grippal et à la grippe confirmée en } \\
\text { laboratoire étaient similaires entre les } \\
\text { différentes interventions (désinfectant } \\
\text { pour les mains : } 0,144 ; \text { désinfectant } \\
\text { pour les mains et masque facial : } 0,124 ; \\
\text { intervention témoin : } 0,137 \text { ). } \\
\text { Si on ne tient compte que du syndrome } \\
\text { grippal et de la grippe confirmée en } \\
\text { laboratoire, les TAS étaient similaires } \\
\text { entre les différentes interventions } \\
\text { (désinfectant pour les mains : } 0,020 ; \\
\text { désinfectant pour les mains et masque } \\
\text { facial : } 0,018 ; \text { intervention témoin : } 0,023 \text { ) }\end{array}$ \\
\hline
\end{tabular}


Tableau 2 (suite): Résumé des données relatives à l'efficacité des pratiques d'hygiène des mains dans la prévention de la transmission de la grippe présumée ou confirmée en laboratoire en milieu communautaire

\begin{tabular}{|c|c|c|c|c|}
\hline Étude & $\begin{array}{c}\text { Taille de } \\
\text { I'échantillon (n) }\end{array}$ & $\begin{array}{c}\text { Intervention d'hygiène des mains } \\
\text { ou pratique déclarée/intervention } \\
\text { témoin }\end{array}$ & $\begin{array}{l}\text { Principal } \\
\text { paramètre } \\
\text { d'évaluation }\end{array}$ & Principaux résultats pertinents \\
\hline \multicolumn{5}{|c|}{ Essai contrôlé randomisé (suite) } \\
\hline $\begin{array}{l}\text { Ram et al., } 2015 \\
(16)\end{array}$ & $\begin{array}{l}377 \text { ménages } \\
\text { (lavage des mains } \\
: 193 ; \text { intervention } \\
\text { témoin : 184) }\end{array}$ & $\begin{array}{l}\text { Information et promotion relatives au } \\
\text { lavage des mains et mise à disposition } \\
\text { de postes de lavage des mains, avec } \\
\text { savon et eau, après le début de la } \\
\text { maladie chez le cas index } \\
\text { Intervention témoin : Pratique standard }\end{array}$ & $\begin{array}{l}\text { TAS liés au } \\
\text { syndrome grippal } \\
\text { et à la grippe } \\
\text { confirmée en } \\
\text { laboratoire }\end{array}$ & $\begin{array}{l}\text { La différence entre les ménages du } \\
\text { groupe d'intervention et les ménages } \\
\text { témoins quant aux TAS liés au syndrome } \\
\text { grippal }(1,24 ; \text { IC à } 95 \%: 0,93-1,65) \text { et } \\
\text { à la grippe confirmée en laboratoire } \\
(2,40 ; \text { IC à } 95 \%: 0,68-8,47) \text { n'était pas } \\
\text { statistiquement significative }\end{array}$ \\
\hline $\begin{array}{l}\text { Simmerman et al., } \\
2011 \text { (17) }\end{array}$ & $\begin{array}{l}465 \text { ménages } \\
\text { (lavage des } \\
\text { mains : } 155 ; \text { lavage } \\
\text { des mains et } \\
\text { masque facial : 155; } \\
\text { intervention } \\
\text { témoin : 155) }\end{array}$ & $\begin{array}{l}\text { Intervention de lavage des mains: } \\
\text { Information sur le lavage des mains et } \\
\text { distributeur de savon } \\
\text { Intervention de lavage des mains et } \\
\text { masque facial : Information sur le lavage } \\
\text { des mains, distributeur de savon et } \\
\text { masque facial } \\
\text { Intervention témoin : Information sur la } \\
\text { nutrition, l'activité physique et l'abandon } \\
\text { du tabagisme }\end{array}$ & $\begin{array}{l}\text { TAS liés au } \\
\text { syndrome grippal } \\
\text { et à la grippe } \\
\text { confirmée en } \\
\text { laboratoire }\end{array}$ & $\begin{array}{l}\text { Les différences quant aux TAS liés } \\
\text { syndrome grippal entre les diverses } \\
\text { interventions étaient statistiquement } \\
\text { significatives (lavage des mains }: 0,17 ; \\
\text { lavage des mains et masque facial : } \\
0,18 ; \text { comparaison : } 0,09 ; p=0,01 \text { ). } \\
\text { Toutefois, il n'y avait aucune différence } \\
\text { statistiquement significative quant } \\
\text { aux TAS liés à la grippe confirmée } \\
\text { en laboratoire entre les diverses } \\
\text { interventions (lavage des mains }: 0,23 ; \\
\text { lavage des mains et masque facial }: 0,23 ; \\
\text { intervention témoin }: 0,19 ; p=0,63) \text {; } \\
\text { D'autres analyses sur la transmission de } \\
\text { la grippe ont mis en évidence des liens } \\
\text { similaires pour le syndrome et la grippe } \\
\text { confirmée en laboratoire lorsque les } \\
\text { groupes d'intervention ont été comparés } \\
\text { aux groupes témoins }\end{array}$ \\
\hline \multicolumn{5}{|l|}{ Étude de cohortes } \\
\hline $\begin{array}{l}\text { Loustalot et al., } \\
2011 \text { (22) }\end{array}$ & 2030 & $\begin{array}{l}\text { Auto-évaluation ou évaluation par un } \\
\text { proche du comportement d'hygiène des } \\
\text { mains (fréquence du lavage des mains et } \\
\text { emploi d'un désinfectant pour les mains) }\end{array}$ & $\begin{array}{l}\text { Déclaration d'un } \\
\text { syndrome grippal } \\
\text { dans le ménage }\end{array}$ & $\begin{array}{l}\text { Dans les ménages avec au moins un cas } \\
\text { de syndrome grippal, il n'y avait aucune } \\
\text { différence statistiquement significative } \\
\text { quant à la fréquence déclarée du lavage } \\
\text { des mains }(p=0,34) \text { ou de l'emploi d'un } \\
\text { désinfectant pour les mains }(p=0,37) \\
\text { comparativement aux ménages sans } \\
\text { syndrome grippal }\end{array}$ \\
\hline \multicolumn{5}{|l|}{ Étude cas témoins } \\
\hline $\begin{array}{l}\text { Zhang et al., } 2013 \\
\text { (24) }\end{array}$ & $\begin{array}{l}162 \text { ménages } \\
\text { (ménages cas : } 54 ; \\
\text { ménages } \\
\text { témoins : 108) }\end{array}$ & $\begin{array}{l}\text { Auto-déclaration de la fréquence du } \\
\text { lavage des mains }\end{array}$ & $\begin{array}{l}\text { Grippe confirmée } \\
\text { en laboratoire }\end{array}$ & $\begin{array}{l}\text { On a noté un lien statistiquement } \\
\text { significatif entre le lavage des mains } \geq \\
3 \text { fois par jour et une réduction de la } \\
\text { probabilité de transmission de la grippe } \\
\text { A pandémique }(\mathrm{H} 1 \mathrm{~N} 1) \text { dans les ménages } \\
\text { (RC: } 0,71 ; \text { IC à } 95 \%: 0,48-0,94)\end{array}$ \\
\hline
\end{tabular}

Abréviations : IC, intervalle de confiance; IVRS, infection des voies respiratoires supérieures; $\mathrm{n}$, nombre; RC, rapport de cotes; TAS, taux d'attaques secondaires; $\geq$, au moins

\section{Discussion}

Dans le présent examen systématique, nous avons repéré 16 études qui ont évalué les répercussions du lavage des mains en tant qu'intervention ou pratique sur la grippe ou sa transmission en milieu communautaire. Les deux tiers de ces études laissent penser que les pratiques d'hygiène des mains pourraient contribuer à prévenir la grippe. Dans la plupart des cas, toutefois, les résultats des études ayant examiné la transmission de la grippe n'étaient pas significatifs sur le plan statistique. La majorité des études présentaient des éléments méthodologiques qui étaient associés à un risque de biais. Les méthodologies employées étaient trop hétérogènes pour qu'il soit possible de réaliser une méta-analyse des études. Nos résultats sont semblables à ceux de deux autres examens systématiques menés sur le sujet, malgré des différences méthodologiques dans la sélection des études. Alors que nous avons recensé des études positives et des études négatives, l'examen effectué par Wong et ses collaborateurs (6) a mis en évidence que l'intervention d'hygiène des mains seule 
n'était pas efficace contre la grippe confirmée en laboratoire, et l'examen de Warren-Gash et ses collaborateurs (7) a fait état de données favorisant une réduction du risque de grippe avec les interventions d'hygiène des mains, selon le contexte communautaire. Par ailleurs, Warren-Gash et ses collaborateurs n'ont relevé aucune donnée confirmant l'efficacité de l'hygiène des mains sur la transmission secondaire de la grippe dans les ménages où il y avait déjà un cas index (7).

\section{Limites}

Les résultats de notre examen doivent être interprétés en tenant compte de certaines limites importantes. En règle générale, la majorité des études exploraient des paramètres qui n'étaient pas spécifiques de l'infection par le virus de la grippe, mais concernaient des syndromes grippaux et des affections respiratoires aiguës susceptibles d'avoir été causés par d'autres virus respiratoires. Par ailleurs, les données recueillies dans un milieu à faible revenu (p. ex. les régions rurales du Bangladesh) ne sont pas nécessairement extrapolables aux milieux à revenu élevé, et vice versa. En outre, dans les essais cliniques contrôlés menés dans un milieu à revenu élevé, les pratiques d'hygiène des mains peuvent être très bonnes d'emblée, ce qui peut amenuiser l'écart entre les groupes d'intervention et les groupes témoins, indépendamment de l'intervention d'hygiène des mains. L'efficacité des interventions d'hygiène des mains dépend aussi du mode de transmission de la grippe et peut être atténuée lorsque la transmission ne se fait pas par contact. La portée du présent examen se limitait aux interventions d'hygiène des mains, indépendamment des autres mesures de santé publique; par conséquent, ces interventions peuvent ne pas être représentatives des mesures de santé publique à plusieurs volets en situation réelle. Enfin, nous n'avons pas effectué de recherche dans la littérature grise, ce qui signifie que des études pourraient avoir été omises.

Il existe aussi des limites qui sont inhérentes aux deux types d'études. Certains ECR retenus manquaient de puissance statistique $(11,13,14)$. Aucun ECR retenu ne donnait suffisamment de détails sur les interventions d'hygiène des mains afin de permettre de comparer ces interventions aux pratiques exemplaires. Les effets de l'hygiène des mains pourraient avoir été sous-estimés en raison de la possibilité de non-observance de l'intervention ou de la contamination des participants témoins. L'adoption de pratiques efficaces d'hygiène des mains peut prendre plus de temps que la période d'intervention d'un essai clinique. Dans le cas des ECR ayant analysé la transmission de la grippe dans les ménages présentant un cas index, il est possible que l'intervention d'hygiène des mains ait été mise en œuvre trop tard par rapport au cours de la maladie chez le cas index de sorte qu'elle n'ait pas pu prévenir efficacement la transmission au sein du ménage. Dans les études sur les ménages, la protection directe et indirecte conférée par la pratique d'hygiène des mains chez les sujets prédisposés ( $p$. ex. les enfants) ne peut être aisément évaluée en raison du manque de renseignements sur les pratiques d'hygiène des mains recueillies auprès de chaque personne.

Pour les études observationnelles retenues, dans lesquelles les pratiques d'hygiène des mains étaient soit auto-déclarées, soit observées, la mesure de ces pratiques pourrait être influencée par un biais de réponse (p. ex. le biais de désirabilité sociale), d'un biais de rappel ou d'un biais de l'observateur (27). Bien que dans la plupart des études observationnelles on ait recueilli des données sur la fréquence du lavage des mains auto-déclarée, on ne précisait pas si on utilisait des critères pour dénombrer les événements de lavage des mains ni la nature de ces critères; il n'est donc pas possible de distinguer les pratiques optimales et sous-optimales de lavage des mains vis-à-vis de la fréquence globale déclarée de lavage des mains. Les études observationnelles peuvent aussi être sensibles à des facteurs de confusion résiduels, à des biais de sélection ou à d'autres biais susceptibles de compliquer l'interprétation des résultats. Bien que les études transversales retenues aux fins de l'examen aient donné des résultats statistiquement significatifs $(18,20,23)$, le protocole transversal ne peut permettre de déterminer si le comportement $d$ 'hygiène des mains déclaré était présent avant la manifestation de la maladie grippale.

\section{Portée et prochaines étapes}

Ces nombreuses limites inhérentes au corpus existant de données mettent en lumière les difficultés d'effectuer des recherches sur ce sujet dans le contexte communautaire, autant pour les protocoles expérimentaux que pour les protocoles d'observation $(6,7,28)$. L'hygiène des mains est une intervention non effractive, non pharmacologique pour laquelle il n'existe pas d'intervention comparative adéquate (29). Il peut par ailleurs être difficile de réaliser des ECR avec des échantillons adéquats pour établir l'importance relative de l'hygiène des mains (30). En outre, le contexte communautaire peut compliquer la mise en œuvre des interventions et l'évaluation des résultats.

Considérant l'ensemble des données probantes de bonne qualité sur les bienfaits des pratiques d'hygiène des mains dans la prévention et le contrôle des maladies infectieuses en général (31), ainsi que les résultats variables et les limites des études actuelles, il n'existe aucune donnée probante indiquant qu'il faut cesser ces pratiques pour réduire le risque d'infection par le virus de la grippe et sa transmission en milieu communautaire. Les pratiques d'hygiène des mains sont non effractives et restent largement applicables dans la prévention des infections et les interventions de contrôle, tout en ne démontrant aucun effet nuisible.

Il faudrait poursuivre les recherches afin de clarifier si, et dans quelles circonstances, les interventions d'hygiène des mains en milieu communautaire sont efficaces pour prévenir la grippe et sa transmission. 


\section{Conclusion}

Les données probantes disponibles sur l'efficacité de l'hygiène des mains dans la prévention de la grippe et sa transmission sont incohérentes et insuffisantes tant sur le plan qualitatif que quantitatif. Cependant, étant donné l'efficacité des pratiques d'hygiène des mains dans la prévention et le contrôle des maladies infectieuses en général, il n'existe aucune donnée probante indiquant qu'il faut cesser ces pratiques pour réduire le risque d'infection par le virus de la grippe et sa transmission en milieu communautaire.

\section{Déclaration des auteurs}

K. M. - Conceptualisation, méthodes, analyse, rédaction de l'ébauche originale - examen et révision

K.Y. - Méthodologie, analyse, rédaction - examen et révision M. T. - Analyse, rédaction - examen et révision

S. R. - Analyse, rédaction - examen et révision

R. S. - Méthodes, analyse, rédaction - examen et révision

L. Z. - Conceptualisation, méthodologie, analyse, rédaction examen et révision

\section{Conflit d'intérêts}

Aucun.

\section{Remerciements}

Les auteurs souhaitent remercier les membres du Groupe de travail sur la préparation du Canada en cas de grippe pandémique pour leurs précieux commentaires. Les auteurs souhaitent aussi souligner la participation de Stella Chen, Lindsay Colas, Lisa Glandon, Kevin Leung, Lisa Paddle, Sharon Smith et Jill Williams à l'examen systématique.

\section{Financement}

Ce travail a été réalisé grâce au soutien de l'Agence de la santé publique du Canada.

\section{Références}

1. Grayson ML, Melvani S, Druce J, Barr IG, Ballard SA, Johnson PD, Mastorakos T, Birch C. Efficacy of soap and water and alcohol-based hand-rub preparations against live $\mathrm{H} 1 \mathrm{~N} 1$ influenza virus on the hands of human volunteers. Clin Infect Dis 2009 Feb;48(3):285-91. DOI PubMed

2. Larson EL, Cohen B, Baxter KA. Analysis of alcohol-based hand sanitizer delivery systems: efficacy of foam, gel, and wipes against influenza $A(H 1 N 1)$ virus on hands. Am J Infect Control 2012 Nov;40(9):806-9. DOl PubMed
3. Allegranzi B, Pittet D. Role of hand hygiene in healthcare-associated infection prevention. J Hosp Infect 2009 Dec;73(4):305-15. DOI PubMed

4. Aiello AE, Coulborn RM, Perez V, Larson EL. Effect of hand hygiene on infectious disease risk in the community setting: a meta-analysis. Am J Public Health 2008 Aug;98(8):1372-81. DOI PubMed

5. Zivich PN, Gancz AS, Aiello AE. Effect of hand hygiene on infectious diseases in the office workplace: A systematic review. Am J Infect Control 2018 Apr;46(4):448-55. DOI PubMed

6. Wong VW, Cowling BJ, Aiello AE. Hand hygiene and risk of influenza virus infections in the community: a systematic review and meta-analysis. Epidemiol Infect 2014 May;142(5):922-32. DOl PubMed

7. Warren-Gash C, Fragaszy E, Hayward AC. Hand hygiene to reduce community transmission of influenza and acute respiratory tract infection: a systematic review. Influenza Other Respir Viruses 2013 Sep;7(5):738-49. DOI PubMed

8. Centre de lutte contre les maladies transmissibles et les infections. Pratiques en matière d'hygiène des mains dans les milieux de soins. Ottawa (ON): Agence de la santé publique du Canada; 2012. http://publications.gc.ca/collections/ collection_2012/aspc-phac/HP40-74-2012-fra.pdf

9. Higgins JP, Altman DG, Gøtzsche PC, Jüni $P$, Moher D, Oxman AD, Savovic J, Schulz KF, Weeks L, Sterne JA; Cochrane Bias Methods Group; Cochrane Statistical Methods Group. The Cochrane Collaboration's tool for assessing risk of bias in randomised trials. BMJ 2011 Oct;343:d5928. DOI PubMed

10. Armijo-Olivo S, Stiles CR, Hagen NA, Biondo PD, Cummings GG. Assessment of study quality for systematic reviews: a comparison of the Cochrane Collaboration Risk of Bias Tool and the Effective Public Health Practice Project Quality Assessment Tool: methodological research. J Eval Clin Pract 2012 Feb;18(1):12-8. DOl PubMed

11. Cowling BJ, Fung RO, Cheng CK, Fang VJ, Chan KH, Seto WH, Yung R, Chiu B, Lee P, Uyeki TM, Houck PM, Peiris JS, Leung GM. Preliminary findings of a randomized trial of non-pharmaceutical interventions to prevent influenza transmission in households. PLoS One 2008 May;3(5):e2101. DOI PubMed

12. Cowling BJ, Chan KH, Fang VJ, Cheng CK, Fung RO, Wai W, Sin J, Seto WH, Yung R, Chu DW, Chiu BC, Lee PW, Chiu MC, Lee HC, Uyeki TM, Houck PM, Peiris JS, Leung GM. Facemasks and hand hygiene to prevent influenza transmission in households: a cluster randomized trial. Ann Intern Med 2009 Oct;151(7):437-46. DOl PubMed

13. Hübner NO, Hübner C, Wodny M, Kampf G, Kramer A. Effectiveness of alcohol-based hand disinfectants in a public administration: impact on health and work performance related to acute respiratory symptoms and diarrhoea. BMC Infect Dis 2010 Aug; 10(1):250. DOI PubMed

14. Larson EL, Ferng YH, Wong-McLoughlin J, Wang S, Haber M, Morse SS. Impact of non-pharmaceutical interventions 
on URIs and influenza in crowded, urban households. Public Health Rep 2010 Mar-Apr;125(2):178-91. DOI PubMed

15. Little P, Stuart B, Hobbs FD, Moore M, Barnett J, Popoola D, Middleton K, Kelly J, Mullee M, Raftery J, Yao G, Carman W, Fleming D, Stokes-Lampard H, Williamson I, Joseph J, Miller $S$, Yardley L. An internet-delivered handwashing intervention to modify influenza-like illness and respiratory infection transmission (PRIMIT): a primary care randomised trial. Lancet 2015 Oct;386(10004):1631-9. DOl PubMed

16. Ram PK, DiVita MA, Khatun-e-Jannat K, Islam M, Krytus K, Cercone E, Sohel BM, Ahmed M, Rahman AM, Rahman M, Yu J, Brooks WA, Azziz-Baumgartner E, Fry AM, Luby SP. Impact of intensive handwashing promotion on secondary household influenza-like illness in rural Bangladesh: findings from a randomized controlled trial. PLoS One 2015 Jun;10(6):e0125200. DOI PubMed

17. Simmerman JM, Suntarattiwong $P$, Levy J, Jarman RG, Kaewchana S, Gibbons RV, Cowling BJ, Sanasuttipun W, Maloney SA, Uyeki TM, Kamimoto L, Chotipitayasunondh T. Findings from a household randomized controlled trial of hand washing and face masks to reduce influenza transmission in Bangkok, Thailand. Influenza Other Respir Viruses 2011 Jul;5(4):256-67. DOI PubMed

18. Adesanya OA, Chiao C. A multilevel analysis of lifestyle variations in symptoms of acute respiratory infection among young children under five in Nigeria. BMC Public Health 2016 Aug;16(1):880. DOI PubMed

19. Doshi S, Silk BJ, Dutt D, Ahmed M, Cohen AL, Taylor TH, Brooks WA, Goswami D, Luby SP, Fry AM, Ram PK. Household-level risk factors for influenza among young children in Dhaka, Bangladesh: a case-control study. Trop Med Int Health 2015 Jun;20(6):719-29. DOI PubMed

20. Hashim S, Ayub ZN, Mohamed Z, Hasan H, Harun A, Ismail N, Rahman ZA, Suraiya S, Naing NN, Aziz AA. The prevalence and preventive measures of the respiratory illness among Malaysian pilgrims in 2013 Hajj season. J Travel Med 2016 Feb;23(2):tav019. DOI PubMed

21. Liu M, Ou J, Zhang L, Shen X, Hong R, Ma H, Zhu BP, Fontaine RE. Protective effect of hand-washing and good hygienic habits against seasonal influenza: a case-control study. Medicine (Baltimore) 2016 Mar;95(11):e3046. DOI PubMed

22. Loustalot F, Silk BJ, Gaither A, Shim T, Lamias M, Dawood F, Morgan OW, Fishbein D, Guerra S, Verani JR, Carlson SA, Fonseca VP, Olsen SJ. Household transmission of 2009 pandemic influenza $A(\mathrm{H} 1 \mathrm{~N} 1)$ and nonpharmaceutical interventions among households of high school students in San Antonio, Texas. Clin Infect Dis 2011 Jan;52 Suppl 1:S146-53. DOI PubMed

23. Wu S, Ma C, Yang Z, Yang P, Chu Y, Zhang H, Li H, Hua W, Tang Y, Li C, Wang $Q$. Hygiene behaviors associated with influenza-like illness among adults in Beijing, China: A large, population-based survey. PLoS One 2016 Feb;11(2):e0148448. DOI PubMed

24. Zhang D, Liu W, Yang P, Zhang Y, Li X, Germ KE, Tang S, Sun W, Wang $\mathrm{Q}$. Factors associated with household transmission of pandemic (H1N1) 2009 among self-quarantined patients in Beijing, China. PLoS One 2013 Oct;8(10):e77873. DOI PubMed

25. Merk H, Kühlmann-Berenzon S, Linde A, Nyrén $O$. Associations of hand-washing frequency with incidence of acute respiratory tract infection and influenza-like illness in adults: a population-based study in Sweden. BMC Infect Dis 2014 Sep;14(1):509. DOI PubMed

26. Torner N, Soldevila N, Garcia JJ, Launes C, Godoy P, Castilla J, Domínguez A; CIBERESP Cases and Controls in Pandemic Influenza Working Group, Spain. Effectiveness of non-pharmaceutical measures in preventing pediatric influenza: a case-control study. BMC Public Health 2015 Jun;15:543. DOI PubMed

27. Ram PK, Halder AK, Granger SP, Jones T, Hall P, Hitchcock $D$, Wright R, Nygren B, Islam MS, Molyneaux JW, Luby SP. Is structured observation a valid technique to measure handwashing behavior? Use of acceleration sensors embedded in soap to assess reactivity to structured observation. Am J Trop Med Hyg 2010 Nov;83(5):1070-6. DOI PubMed

28. Jefferson T, Del Mar C, Dooley L, Ferroni E, Al-Ansary LA, Bawazeer GA, van Driel ML, Foxlee R, Rivetti A. Physical interventions to interrupt or reduce the spread of respiratory viruses: systematic review. BMJ 2009 Sep;339:b3675. DOI PubMed

29. Comité consultatif de la médecine tropicale et de la médecine des voyages (CCMTMV). Déclaration sur la Diarrhée du Voyageur. Une déclaration du Comité consultatif (DCC). Ottawa (ON): Agence de la santé publique du Canada; 2015 [mise à jour le 1er mai 2015]. https://www. canada.ca/fr/sante-publique/services/ccmtmv/declarationdiarrhee-voyageur.html

30. Organisation mondiale de la Sánte. Résumé des Recommandations de l'OMS pour l'Hygiène des Mains au cours des Soins: Premier Défi Mondial pour la Sécurité des Patients Un Soin propre est un Soin plus sûr. Genève: Organisation mondiale de la Santé; 2009. http://apps. who.int/iris/bitstream/handle/10665/70469/WHO_IER_ PSP_2009.07_fre.pdf?sequence=1

31. Centers for Disease Control and Prevention. Handwashing: Publications, data, \& statistics. Atlanta (GA): Centers for Disease Control and Prevention; [mise à jour 2015 Jul 22]. https://www.cdc.gov/handwashing/publications-data-stats. html 


\section{Annexe 1 : Stratégie de recherche dans les bases de données électroniques et résultats}

\begin{tabular}{|c|c|c|}
\hline $\begin{array}{l}\text { Numéro } \\
\text { de série }\end{array}$ & Recherches & Résultats \\
\hline \multicolumn{3}{|l|}{ MEDLINE } \\
\hline 1 & hand hygiene/ or hand disinfection/ & 5680 \\
\hline 2 & (hand? adj3 (hygien* or wash* or disinfect* or sanitiz* or antiseptic ${ }^{\star}$ or steriliz* or decontaminat* or clean ${ }^{\star}$ )).tw. & 7433 \\
\hline 3 & handwash*.tw. & 1661 \\
\hline 4 & 1 or 2 or 3 & 10550 \\
\hline 5 & exp residence characteristics/ or exp schools/ or workplace/ or exp "Non-Medical Public and Private Facilities"/ & 280888 \\
\hline 6 & $\begin{array}{l}\text { (communit* or domicile? or domestic or residential or neighborhood? or household? or home? or family or } \\
\text { families or school* or college? or universit* or "education* setting*" or student? or daycare? or childcare or } \\
\text { workplace? or workspace? or worksite? or employee? or "public setting?" or "non healthcare setting*" or "non } \\
\text { health care setting*").tw. }\end{array}$ & 2148929 \\
\hline 7 & ((work or job or public) adj3 (setting? or location? or site? or place?)).tw. & 15472 \\
\hline 8 & 5 or 6 or 7 & 2296190 \\
\hline 9 & influenza, human/ or exp influenzavirus a/ or exp influenzavirus b/ & 63179 \\
\hline 10 & (influenza* or flu or h1n\# or h2n\# or h3n\# or h5n\# or h6n\# or h7n\# or h9n\# or h10n\#).tw. & 110315 \\
\hline 11 & $\begin{array}{l}\text { common cold/ or respiratory tract infections/ or rhinitis/ or sinusitis/ or fever/ or cough/ or pharyngitis/ or } \\
\text { sneezing/ or myalgia/ or headache/ or vomiting/ or diarrhea/ }\end{array}$ & 201878 \\
\hline 12 & $\begin{array}{l}\text { ("common cold" or "respiratory infection*" or "respiratory virus*" or "respiratory tract infection*" or "respiratory } \\
\text { illness*" or fever* or cough* or "sore throat" or "runny nose" or "nasal congestion" or sneezing or malaise* or } \\
\text { myalgia or headache* or "muscle ache*" or vomit" or diarrhea or diarrhoea).tw. }\end{array}$ & 419905 \\
\hline 13 & 9 or 10 or 11 or 12 & 616262 \\
\hline 14 & 4 and 8 and 13 & 717 \\
\hline 15 & limit 14 to (english or french) & 674 \\
\hline 16 & 15 and "Editorial" [Publication Type] & 2 \\
\hline 17 & 15 and "Newspaper Article" [Publication Type] & 1 \\
\hline 18 & 15 not $(16$ or 17$)$ & 671 \\
\hline 19 & hand hygiene/ or hand disinfection/ & 5680 \\
\hline 20 & (hand? adj3 (hygien* or wash* or disinfect* or sanitiz* or antiseptic* or steriliz* or decontaminat* or clean*)).tw. & 7433 \\
\hline 21 & handwash*.tw. & 1661 \\
\hline 22 & 19 or 20 or 21 & 10550 \\
\hline 23 & influenza, human/ or exp influenzavirus a/ or exp influenzavirus b/ & 63179 \\
\hline 24 & (influenza* or flu or h1n\# or h2n\# or h3n\# or h5n\# or h6n\# or h7n\# or h9n\# or h10n\#).tw. & 110315 \\
\hline 25 & $\begin{array}{l}\text { common cold/ or respiratory tract infections/ or rhinitis/ or sinusitis/ or fever/ or cough/ or pharyngitis/ or } \\
\text { sneezing/ or myalgia/ or headache/ or vomiting/ or diarrhea/ }\end{array}$ & 201878 \\
\hline 26 & $\begin{array}{l}\text { ("common cold" or "respiratory infection*" or "respiratory virus*" or "respiratory tract infection*" or "respiratory } \\
\text { illness*" or fever* or cough* or "sore throat" or "runny nose" or "nasal congestion" or sneezing or malaise* or } \\
\text { myalgia or headache* or "muscle ache*" or vomit" or diarrhea or diarrhoea).tw. }\end{array}$ & 419905 \\
\hline 27 & 23 or 24 or 25 or 26 & 616262 \\
\hline 28 & 22 and 27 & 1349 \\
\hline 29 & limit 28 to (english or french) & 1249 \\
\hline 30 & 29 and "Editorial" [Publication Type] & 15 \\
\hline 31 & 29 and "Newspaper Article" [Publication Type] & 3 \\
\hline 32 & 29 and "Comment" [Publication Type] & 32 \\
\hline 33 & 29 not (30 or 31 or 32$)$ & 1203 \\
\hline 34 & 33 not 18 & 538 \\
\hline
\end{tabular}


Annexe 1 (suite) : Stratégie de recherche dans les bases de données électroniques et résultats

\begin{tabular}{|c|c|c|}
\hline $\begin{array}{l}\text { Numéro } \\
\text { de série }\end{array}$ & Recherches & Résultats \\
\hline \multicolumn{3}{|l|}{ Embase } \\
\hline 1 & hand washing/ or hand disinfection/ & 11298 \\
\hline 2 & (hand? adj3 (hygien* or wash* or disinfect* or sanitiz* or antiseptic* or steriliz* or decontaminat* or clean*)).tw. & 10307 \\
\hline 3 & handwash*.tw. & 1863 \\
\hline 4 & 1 or 2 or 3 & 16007 \\
\hline 5 & community/ or community living/ or household/ or home/ or exp school/ or workplace/ or building/ & 456912 \\
\hline 6 & $\begin{array}{l}\text { (communit* or domicile? or domestic or residential or neighborhood? or household? or home? or family or } \\
\text { families or school* or college? or universit* or "education* setting*" or student? or daycare? or childcare or } \\
\text { workplace? or workspace? or worksite? or employee? or "public setting?" or "non healthcare setting*" or "non } \\
\text { health care setting*").tw. }\end{array}$ & 2757553 \\
\hline 7 & ((work or job or public) adj3 (setting? or location? or site? or place?)).tw. & 19320 \\
\hline 8 & 5 or 6 or 7 & 2899020 \\
\hline 9 & exp influenza/ or exp influenza virus/ & 88859 \\
\hline 10 & (influenza* or flu or h1n\# or h2n\# or h3n\# or h5n\# or h6n\# or h7n\# or h9n\# or h10n\#).tw. & 126819 \\
\hline 11 & $\begin{array}{l}\text { common cold/ or respiratory tract infection/ or fever/ or rhinitis/ or sinusitis/ or coughing/ or sore throat/ or } \\
\text { rhinorrhea/ or nose obstruction/ or pharyngitis/ or sneezing/ or myalgia/ or headache/ or vomiting/ or diarrhea/ }\end{array}$ & 737993 \\
\hline 12 & $\begin{array}{l}\text { "common cold" or "respiratory infection*" or "respiratory virus*" or "respiratory tract infection*" or "respiratory } \\
\text { illness*" or fever or cough* or "sore throat" or "runny nose" or "nasal congestion" or sneezing or malaise* or } \\
\text { myalgia or headache* or "muscle ache*" or vomit* or diarrhea or diarrhoea).tw. }\end{array}$ & 562610 \\
\hline 13 & 9 or 10 or 11 or 12 & 1087580 \\
\hline 14 & 4 and 8 and 13 & 1092 \\
\hline 15 & limit 14 to (english or french) & 1041 \\
\hline 16 & 15 and "Editorial" [Publication Type] & 6 \\
\hline 17 & 15 not 16 & 1035 \\
\hline 18 & hand washing/ or hand disinfection/ & 11298 \\
\hline 19 & (hand? adj3 (hygien* or wash* or disinfect* or sanitiz* or antiseptic* or steriliz* or decontaminat* or clean*)).tw. & 10307 \\
\hline 20 & handwash*.tw. & 1863 \\
\hline 21 & 18 or 19 or 20 & 16007 \\
\hline 22 & exp influenza/ or exp influenza virus/ & 88859 \\
\hline 23 & (influenza* or flu or h1n\# or h2n\# or h3n\# or h5n\# or h6n\# or h7n\# or h9n\# or h10n\#).tw. & 126819 \\
\hline 24 & $\begin{array}{l}\text { common cold/ or respiratory tract infection/ or fever/ or rhinitis/ or sinusitis/ or coughing/ or sore throat/ or } \\
\text { rhinorrhea/ or nose obstruction/ or pharyngitis/ or sneezing/ or myalgia/ or headache/ or vomiting/ or diarrhea/ }\end{array}$ & 737993 \\
\hline 25 & $\begin{array}{l}\text { ("common cold" or "respiratory infection*" or "respiratory virus*" or "respiratory tract infection*" or "respiratory } \\
\text { illness*" or fever or cough* or "sore throat" or "runny nose" or "nasal congestion" or sneezing or malaise* or } \\
\text { myalgia or headache* or "muscle ache*" or vomit* or diarrhea or diarrhoea).tw. }\end{array}$ & 562610 \\
\hline 26 & 22 or 23 or 24 or 25 & 1087580 \\
\hline 27 & 21 and 26 & 2512 \\
\hline 28 & limit 27 to (english or french) & 2370 \\
\hline 29 & 28 and "Editorial" [Publication Type] & 68 \\
\hline 30 & 28 not 29 & 2302 \\
\hline 31 & 30 not 17 & 1267 \\
\hline
\end{tabular}




\section{Annexe 1 (suite) : Stratégie de recherche dans les bases de données électroniques et résultats}

\begin{tabular}{|c|c|c|}
\hline $\begin{array}{l}\text { Numéro } \\
\text { de série }\end{array}$ & Recherches & Résultats \\
\hline \multicolumn{3}{|c|}{ Bibliothèque Cochrane } \\
\hline 1 & [mh $\wedge$ "hand hygiene"] or [mh $\wedge$ "hand disinfection"] & 363 \\
\hline 2 & $\begin{array}{l}\text { (hand? near/3 (hygien* or wash* or disinfect* or sanitiz* or antiseptic* or steriliz* or decontaminat* or } \\
\text { clean*)):ti,ab,kw }\end{array}$ & 154 \\
\hline 3 & handwash*:ti,ab,kw & 217 \\
\hline 4 & 1 or 2 or 3 & 544 \\
\hline 5 & $\begin{array}{l}\text { [mh ^ "residence characteristics"] or [mh schools] or [mh ^ workplace] or [mh "Non-Medical Public and Private } \\
\text { Facilities"] }\end{array}$ & 3578 \\
\hline 6 & $\begin{array}{l}\text { (communit* or domicile? or domestic or residential or neighborhood? or household? or home? or family or } \\
\text { families or school* or college? or universit* or (education* next setting*) or student? or daycare? or childcare or } \\
\text { workplace? or workspace? or worksite? or employee? or (public next setting?) or "non healthcare setting" or "non } \\
\text { health care setting" or "non healthcare settings" or "non health care settings"):ti,ab,kw }\end{array}$ & 101164 \\
\hline 7 & ((work or job or public) near/3 (setting? or location? or site? or place?)):ti,ab,kw & 248 \\
\hline 8 & 5 or 6 or 7 & 101724 \\
\hline 9 & [mh $\wedge^{\wedge}$ influenza, human"] or [mh "influenzavirus a"] or [mh "influenzavirus b"] & 1830 \\
\hline 10 & (influenza* or flu or h1n? or h2n? or h3n? or h5n? or h6n? or h7n? or h9n? or h10n?):ti,ab,kw & 7611 \\
\hline 11 & 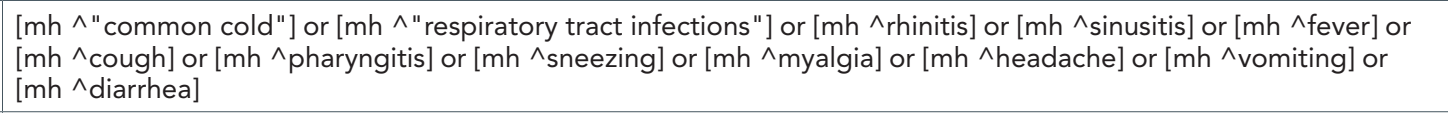 & 13353 \\
\hline 12 & $\begin{array}{l}\text { ("common cold" or (respiratory next infection*) or (respiratory next virus*) or (respiratory next tract next } \\
\text { infection*) or (respiratory next illness*) or fever }{ }^{\star} \text { or cough* or "sore throat" or "runny nose" or "nasal } \\
\text { congestion" or sneezing or malaise* or myalgia or headache* or (muscle next ache*) or vomit* or diarrhea or } \\
\text { diarrhoea):ti,ab,kw }\end{array}$ & 77363 \\
\hline 13 & 9 or 10 or 11 or 12 & 82910 \\
\hline 14 & 4 and 8 and 13 & 86 \\
\hline 15 & [mh $\wedge$ "hand hygiene"] or [mh $\wedge$ "hand disinfection"] & 363 \\
\hline 16 & $\begin{array}{l}\text { (hand? near/3 (hygien* or wash* or disinfect* or sanitiz* or antiseptic* or steriliz* or decontaminat* or } \\
\text { clean*)):ti,ab,kw }\end{array}$ & 154 \\
\hline 17 & handwash*:ti,ab,kw & 217 \\
\hline 18 & 15 or 16 or 17 & 544 \\
\hline 19 & [mh ^ "influenza, human"] or [mh "influenzavirus a"] or [mh "influenzavirus b"] & 1830 \\
\hline 20 & (influenza* or flu or h1n? or h2n? or h3n? or h5n? or h6n? or h7n? or h9n? or h10n?):ti,ab,kw & 7611 \\
\hline 21 & 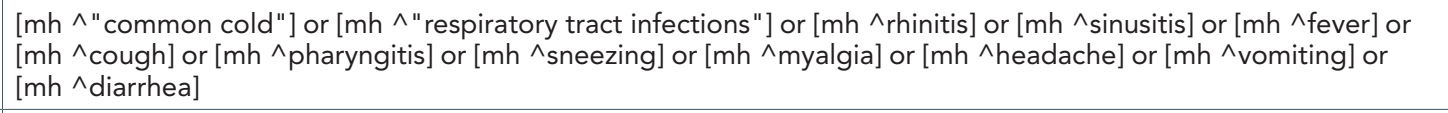 & 13353 \\
\hline 22 & $\begin{array}{l}\text { ("common cold" or (respiratory next infection*) or (respiratory next virus*) or (respiratory next tract next } \\
\text { infection*) or (respiratory next illness*) or fever* or cough* or "sore throat" or "runny nose" or "nasal } \\
\text { congestion" or sneezing or malaise* or myalgia or headache* or (muscle next ache*) or vomit* or diarrhea or } \\
\text { diarrhoea):ti,ab,kw }\end{array}$ & 77363 \\
\hline 23 & 19 or 20 or 21 or 22 & 82910 \\
\hline 24 & 18 and 23 & 127 \\
\hline 25 & 24 not 14 & 41 \\
\hline
\end{tabular}

